\title{
New Improvements to Millimeter-Wave Body Scanners
}

\author{
Douglas MCMAKIN*, David SHEEN, Thomas HALL, Jonathan TEDESCHI, A. Mark JONES \\ Pacific Northwest National Laboratory, Operated for the U. S. Department of Energy \\ by Battelle Memorial Institute, Richland, WA, USA \\ DOI: $10.15221 / 17.263$ http://dx.doi.org/10.15221/17.263
}

\begin{abstract}
The Pacific Northwest National Laboratory (PNNL) pioneered the development of the millimeter-wave body scanner in the 1990s. PNNL licensed this technology to companies in the security and apparel industries. Millimeter-wave body scanners are ideally suited for applications in high-traffic areas such as airports and retail spaces because the scanners are extremely fast and do not require people to remove outer garments. Since low-power millimeter-waves are non-ionizing, the scanners present no health hazards during scanning operations. The resolution of the resulting imagery enables highly accurate threat detection (for security) and body measurement (for apparel). PNNL is actively engaged in the research and development of methods to continue to improve this technology. This paper will discuss the underlying millimeter-wave scanner technology and new developments that improve the image quality of these systems. New developments in the use of millimeter wave scanning for accurate location identification in radiation therapy may also be utilized.
\end{abstract}

Keywords: Millimeter waves, body measurements, personnel screening, synthetic radar imaging, polarimetric imaging

\section{Introduction}

Over last decade, millimeter-wave body scanners have emerged out of the research laboratory and entered the security and apparel marketplaces. These scanners are used daily at airport checkpoints and by apparel merchants. These scanners offer a number of important benefits to their stakeholders. For one, they can rapidly scan fully clothed people in one to ten seconds to determine if they are either carrying concealed threats or obtain an accurate three-dimensional digital body. In both cases, the privacy concerns are eliminated either by using privacy algorithm software to remove human features [1] or utilizing Health insurance Portability and Accountability Act (HIPAA) administrative controls for apparel uses [2]. Another important benefit is that this technology is non-harmful to people because low-powered millimeter waves are non-ionizing [3, 4].

Research laboratories and product developers have developed a number of millimeter-wave body scanners over the last 20 years $[5,6]$. Most product developers have focused on security applications. In general, there are two types of millimeter-wave body scanners - passive or active.

Passive millimeter-wave (PMMW) body scanners are similar in nature to Infrared (IR) cameras except that they utilize the naturally occurring millimeter-waves that are emitted from the body and concealed threat or object hidden in the clothing instead of Infrared emissions. Passive millimeter-wave imagery is usually formed with a quasi-optical focusing element - similar to camera lenses but usually physically much larger. PMMW body scanners form only two-dimensional imagery [7]. On the other hand, active millimeter-wave (AMMW) body scanners illuminate the person with very low-power millimeter waves. AMMW body scanners can form three-dimensional imagery because they utilize either wide bandwidth synthetic focusing techniques or they use a variable reflective focusing lens that can rapidly focus over a volume. There are a number of active millimeter-wave body scanners that have been developed over the last decade, but only one that has wide spread commercial deployment in airports and apparel shopping malls - the PNNL millimeter-wave body scanner. This paper will focus on the PNNL millimeter wave body scanner technology, imaging results, new technology that is emerging from the laboratory, and potential new application for this technology in radiation therapy.

\footnotetext{
* doug.memakin@pnnl.gov, +1-509-375-2206, www.pnnl.gov
} 


\section{Background}

PNNL began developing the millimeter-wave body scanner back in the early 1990s for the Federal Aviation Administration (FAA). Back then, the FAA, the forerunner of the TSA, was searching for new non-harmful technology to screen people for emerging non-metallic threats such as handguns fabricated out of composite materials and explosives made from plastics and liquids. Millimeter-wave technology was a good choice because their wavelengths were small enough to form high resolution imagery and they could readily penetrate through clothing barriers. Additionally, at very low power levels, they were not harmful. Furthermore, synthetic aperture focusing techniques could be utilized with millimeter-wave technology to form high-resolution diffraction limited imagery that allowed for a variety of scanning configurations. That is to say, physical focusing elements such as quasi-optical reflective surfaces were not needed because the image formation was performed in a computer with digital synthetic focusing techniques. If, on the other hand, physical focusing elements were used, generally the aperture sized would be constrained, which would limit coverage (field-of-view) and image resolution.

The FAA funded PNNL to develop millimeter-wave body scanning technology. PNNL developed a number of systems using a couple of scanning configurations - planer and cylindrical. In 2000, PNNL realized that not only could the millimeter-wave body scanning technology be used for security applications, but it could also be used to obtain accurate three-dimensional digital body measurements without a person disrobing. This ability to rapidly scan people in street clothing differentiated it from both optical and laser body scanners. This capability allowed it to be deploy in high traffic retail locations such as malls and apparel stores. PNNL captured the intellectual property on the millimeter-wave body scanners and licensed it to two companies in two diverse market sectors - security and apparel. These companies, L-3 Communications and Unique Solutions, have commercialized this technology and deployed the first generation technology into their respective venues. Figure 1 shows the L-3 Communication's ProVision ATD security scanner and Unique Solution's Me-Ality 3-D digital body measurement scanners.
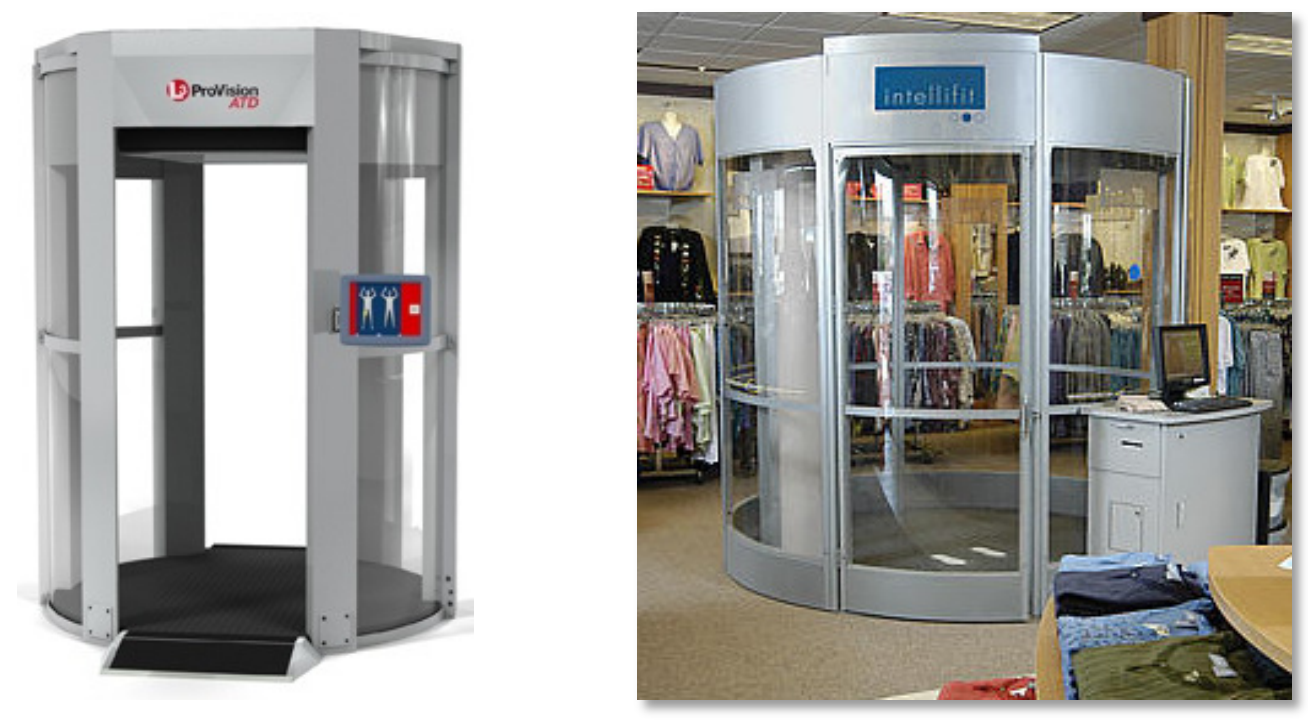

Fig. 1. L-3 Communication's ProVision security scanner (left) and Unique Solution's Me-Ality 3-D digital body measurement scanner (right).

PNNL continues to develop and improve this technology. We have new innovation that will improve the imaging resolution both in depth and laterally. Additionally, we have new ideas to improve body coverage to reduce holes in the data and reduce imaging artifacts. PNNL is presently fabricating the next generation millimeter-wave body scanner prototype for the Department of Homeland Security. The technology developed in this project could be used for both security and body measurement applications. This paper will detail the millimeter-wave body scanning technology and new technical improvements that are under development. 


\section{Millimeter-wave body scanning technology}

In many ways, the millimeter-wave body scanner operates like many other imaging based systems. For example, most imaging systems require that the scene be illuminated with some form of electromagnetic waves (optical, infrared, etc.). The imaging system must be able to capture the reflected electromagnetic signals from the scene and the imaging system must also be able to focus the electromagnetic waves to form the imagery and display them to the user. Millimeter-wave body scanners use these functions to form imaging. Instead of optical electromagnetic waves, millimeterwaves are used. Instead of using optical lighting to illuminate the scene and CCD sensor reflected signals, a linear antenna array coupled to the coherent millimeter-wave transceiver is used to both illuminate the scene and received reflected signals from the scene. The transceiver provides time of flight information that can be used to form three dimensional imagery The linear array is used as one dimension of the imaging aperture and a mechanical scanner (planer or cylindrical) is used to move the array which forms the second dimension of the aperture. A synthetic aperture image reconstruction algorithm is used to focus the digitized reflect data from the array into to image data set [8 - 12].

\subsection{Millimeter-waves}

Millimeter-waves are electromagnetic waves in the $30-300 \mathrm{GHz}$ range with wavelengths that range from $1-10$ millimeters. PNNL also uses electromagnetic waves from $3-30 \mathrm{GHz}$, which is generally considered to be the microwave part of the electromagnetic spectrum. The wavelengths in the microwave spectrum range from $10-100$ millimeters. Both millimeter-waves and microwaves readily penetrate low-dielectric optical opaque barriers such as clothing. However, lower microwave and millimeter-wave frequencies are better at penetrating layered clothing because their wavelengths are longer. Additionally, at lower microwave and millimeter-wave frequencies, the clothing is less reflective, which reduces clutter in the imagery. However, because the wavelengths are long compared to Infrared and optical frequencies, the millimeter-wave scanner imaging aperture most be much larger to produce high resolution images. Millimeter-waves do not penetrate skin. Therefore, the millimeter-wave arrays must scan both the front and back of the person to obtain full coverage.

\subsection{Millimeter-wave Scanning Technology}

PNNL has developed several millimeter-wave imaging configuration. One uses a planar aperture and the other uses a cylindrical reconstruction. Both configurations scan transceiver or transceiver- arrays over an aperture to illuminate the scene and receive reflected signals for the target under investigation. The transceivers can be a single antenna pair, a linear array, or a two-dimensional array. The two configurations are shown in Figure 2. The authors have written extensively on this papers describes the various configurations.
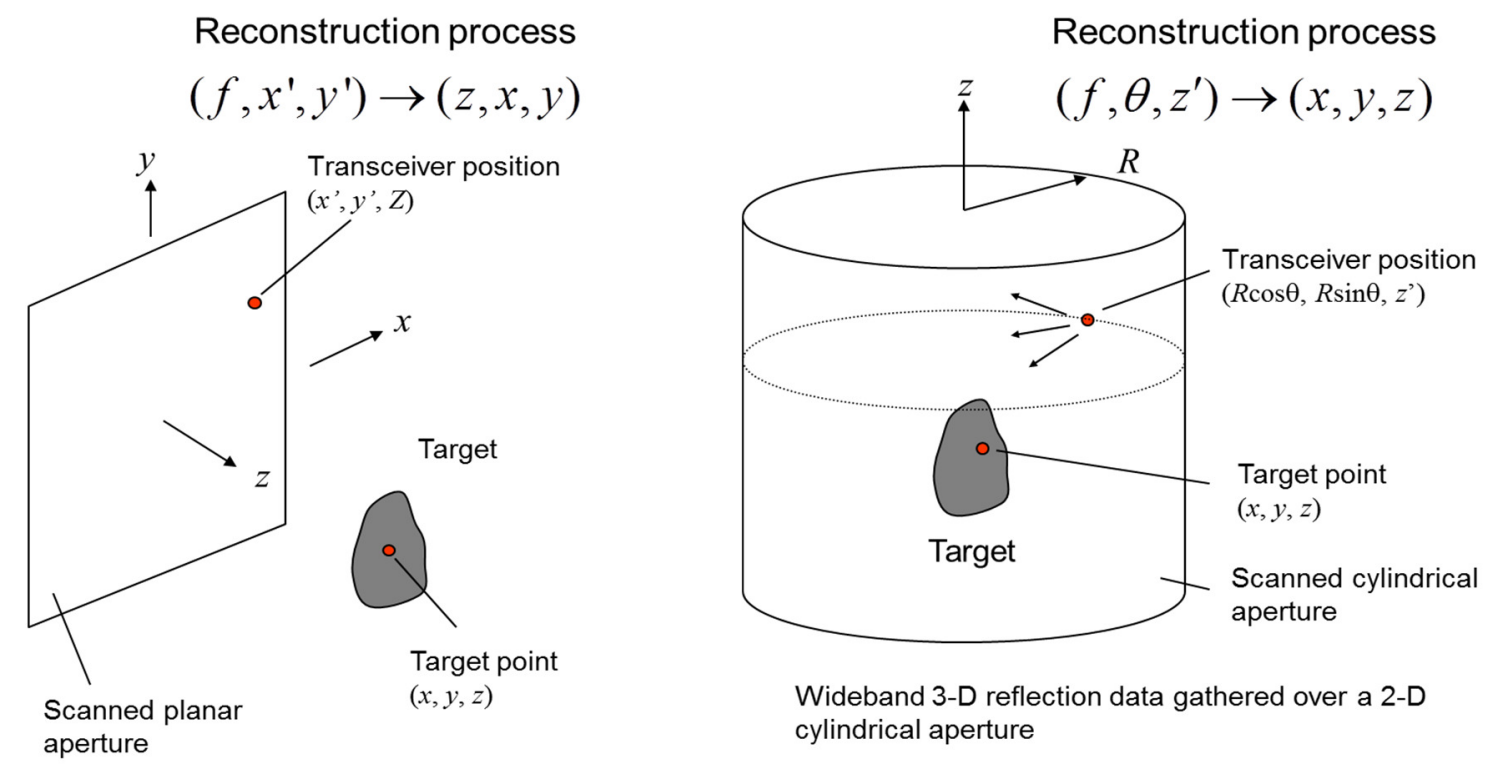

Fig. 2. Planer and cylindrical scanning configureations. 
Generally speaking, linear arrays are used for both configurations because they allow fast mechanical scanning and the ability to freeze motion. Linear arrays provide very good system performance and are less expensive than two-dimensional arrays.

\subsection{Imaging Results}

Figure 3 shows the flow chart of the screening methodology used for biometric identification of the humans using synthetic millimeter-wave imaging techniques. First, millimeter-wave scanner data of a person are collected over a planer or cylindrical aperture. Second, the scanner data is reconstructed into an image using synthetic aperture reconstruction techniques. Third, depending on the application, 3D surface definition files are generated, which include point cloud, mesh, surface renderings or silhouette (security). Finally, application software can utilize 3D surface synthesis surface definition files. For example, automatic threat detection software can be used to detect concealed threats for security applications and body measurement software can be used to determine the 3-D measurements of the human body [13].

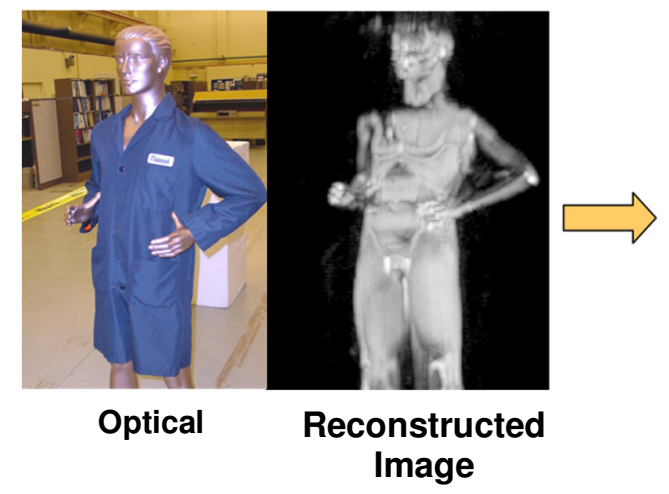

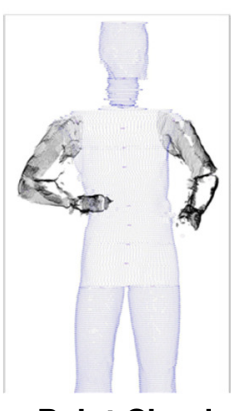

Point Cloud

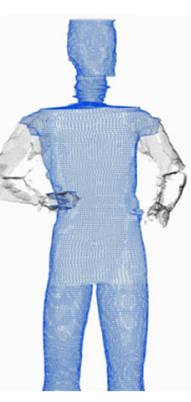

Mesh

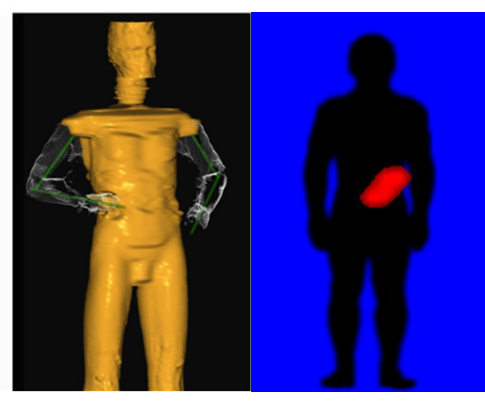

Surface
Threat Superimposed on

Generic

Fig. 3. Screening methodology flow chart.

Figure 4 shows the progression from the combined cylindrical holographic images to the development of the various surface files.

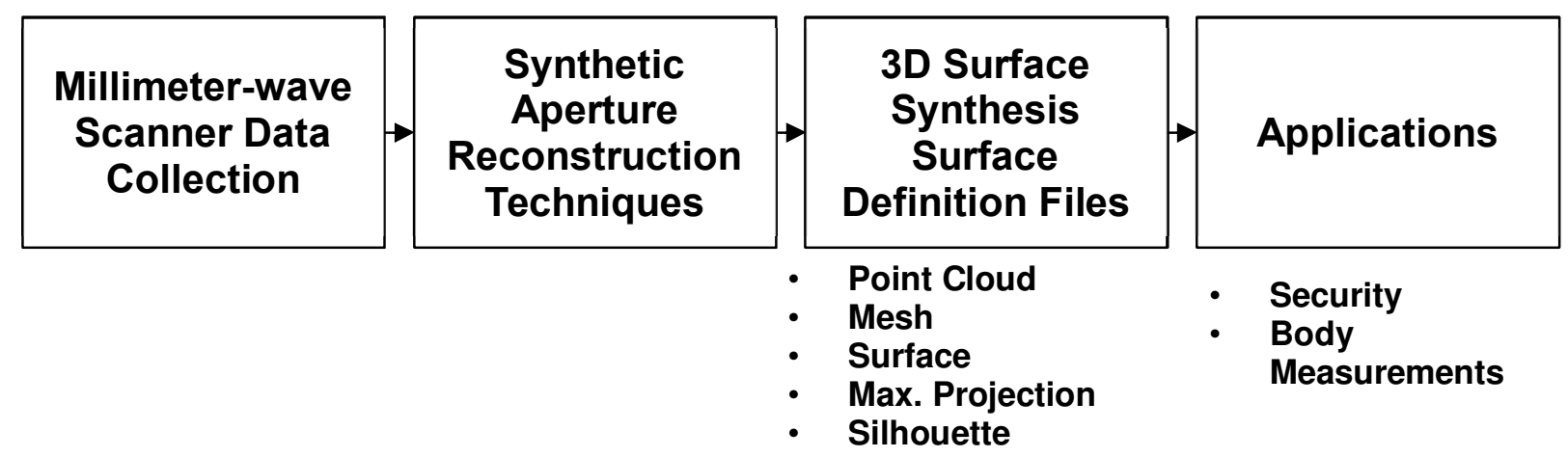

Fig. 4. Progression from reconstructed image to surface definition file.

Figure 5 shows laboratory imaging results from of a full sized mannequin using a cylindrical and combined cylindrical synthetic aperture image reconstruction algorithms. These are single frame imaging results from full 360 degree cylindrical scans. Imaging artifacts from body corner traps and crevices can be observed in these images along with dark areas from illumination limitations. 

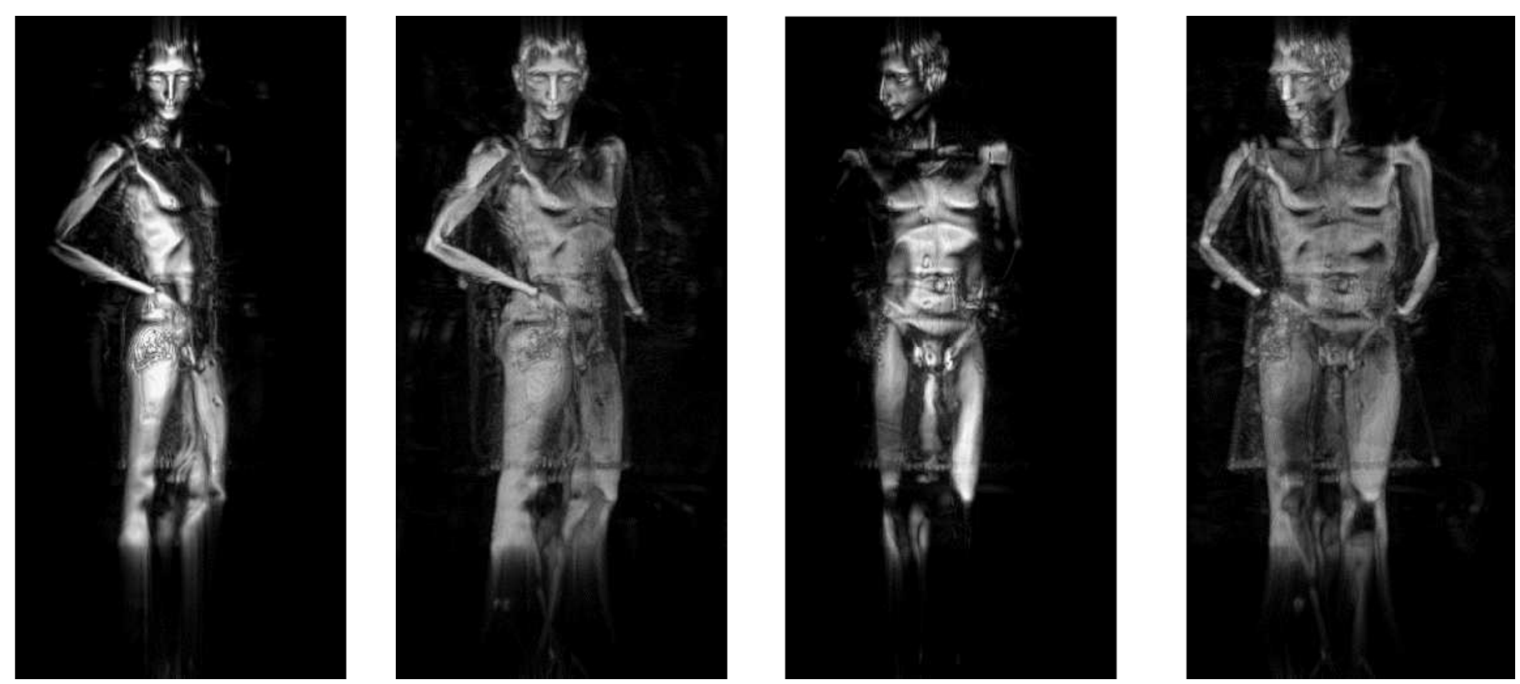

Fig. 5. 24 - $40 \mathrm{GHz}$ cylindrical and combined cylindrical millimeter-wave imagery of a mannequin.

\section{New developments}

PNNL is working on technical improvements to the millimeter-wave scanners that will significantly improve image quality. These technical improvements will improve threat detection performance in security scanners and body measurement accuracy in apparel scanners. The millimeter-wave scanner imaging improvements that PNNL is working on are as follows:

- Improved Depth Resolution

- Improved Lateral Resolution

- Reduced Imaging Artifacts

- Improved Illumination and Coverage

The technical improvements include increasing the transceiver bandwidth to improve depth resolution, increasing the array antenna beamwidth to improve lateral resolution and body coverage (illumination), and utilizing polarization illumination and receiving techniques to reduce imaging artifacts from the body corner traps and crevices. The technology enhancements used to improve millimeter-wave scanner imager are discussed in the following sections.

\subsection{Improved Depth Resolution}

Depth resolution in the millimeter-wave imagery can be improved by increasing the transceiver bandwidth. Depth resolution improvement is a linear function of the bandwidth of the transceiver and is given by

$$
\delta_{z}=\frac{c}{2 B}
$$

Were $c$ is the speed of light and $B$ is the bandwidth.

PNNL has developed a very wide bandwidth transceiver that covers the frequency range from $10-40$ $\mathrm{GHz}$ or $30 \mathrm{GHz}$ of bandwidth. The depth resolution of $30 \mathrm{GHz}$ of bandwidth is $0.5 \mathrm{~cm}$ (defined by resolving two points in space). The simplified $10-40 \mathrm{GHz}$ transceiver design is shown in Figure 6 . This transceiver design utilizes heterodyne up-conversion and down-conversion techniques and produces baseband in-phase (I) and quadrature $(\mathrm{Q})$ signatures. 


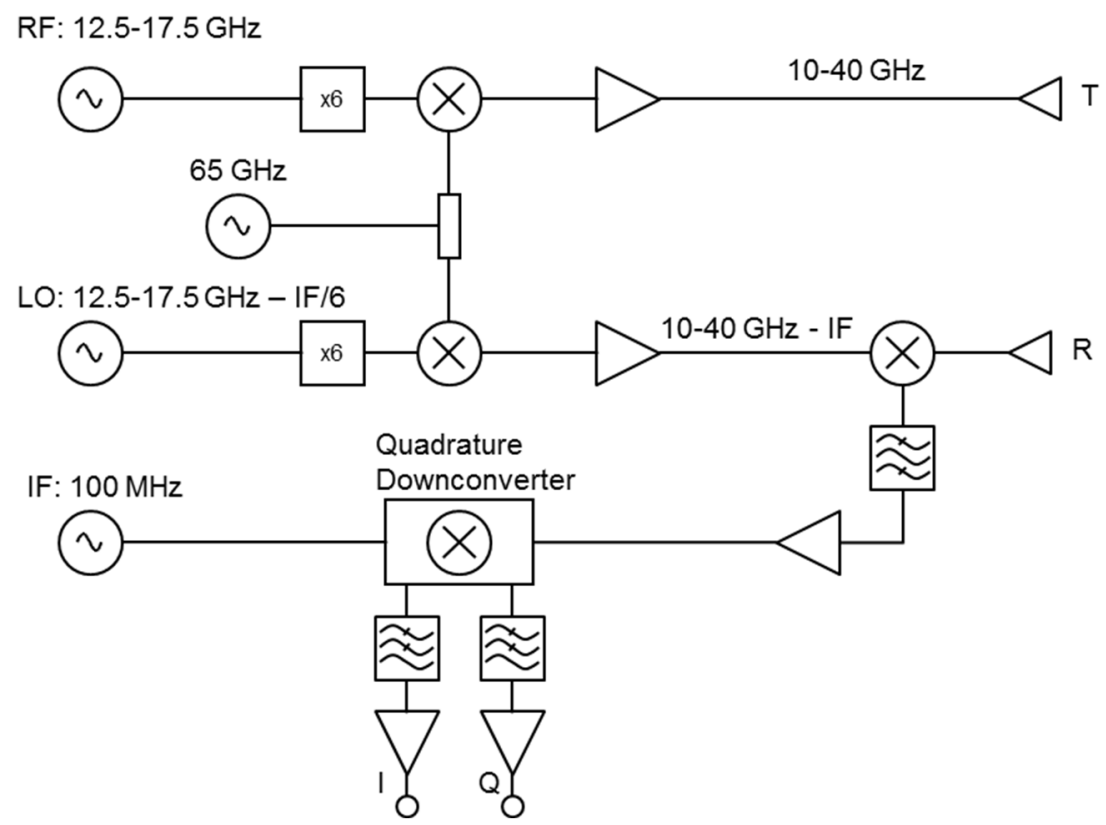

Fig. 6. Simplified 10-40 GHz transceiver.

\subsection{Improved Lateral Resolution}

The lateral resolution obtained from the millimeter-wave scanners is limited by diffraction to approximately,

$$
\delta_{x}=\frac{\lambda_{c}}{4 \sin \left(\theta_{b} / 2\right)}
$$

where $\lambda_{c}$ is the wavelength at the center frequency, $\theta_{b}$ is the antenna beamwidth. For wide-beamwidth operation $\left(\theta_{b} \geq 60^{\circ}\right)$ half-wavelength resolution is readily achievable. PNNL developed very widebeamwidth antennas using fully custom cavity - back dual-arm spiral antennas. The spiral antenna model and optical image is show in Figure 7. These antennas have beamwidths on the order of 90 degrees.
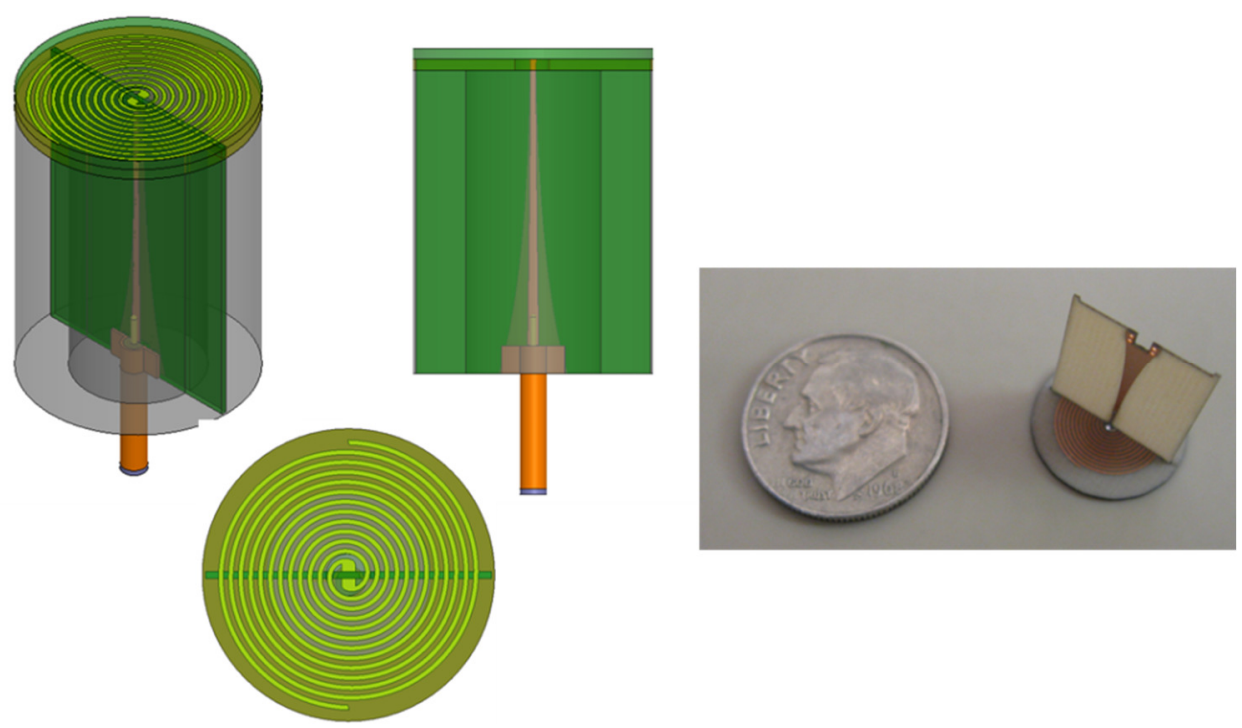

Fig. 7. Spiral $10-40 \mathrm{GHz}$ antenna solid model renderings and a photograph showing the spiral antenna relative to a dime coin. 


\subsection{Polarimetric Information}

The spiral antennas allows for circular polarized illumination and reception in the millimeter-scanners. Both Right-Hand (RCP) and Left-Hand circular polarized (LCP) antennas can be fabricated with the spiral antennas. Imaging artifacts from body corner traps and crevices can be minimized by using circularly cross-polarized imaging configuration. This can be implemented by transmitting with a RCP antenna and receiving with an LCP antennas [14].

\subsection{Improved Illumination Quality}

Another advantage of using wide beamwidth spiral antennas is that they improved illumination coverage over the body. The quality of the illumination can reduces specular reflection from high angular surfaces of the body such as the shoulders. Improved illumination reduces dark areas in the imagery because the wide beamwidth antennas are able to capture the reflected millimeter-wave signals from high angular surfaces [15].

\subsection{Improved Image Results}

Experimental imaging studies were conducted with a full sized mannequin using the improvements discussed above for both planer and cylindrical scanning configurations. In these experiments, the mannequin was completely unclothed. Figure 8 shows a planer $10-40 \mathrm{GHz}$ circularly cross polarized image of a mannequin using the spiral antennas shown above. The planer scan of the mannequin was taken at close range to improve resolution and coverage. This is a three-dimensional image of the mannequin, but the depth information is suppressed using maximum value projection techniques that are used to display the imagery. As shown in Figure 8, resolution is very high, artifacts from body corner traps are minimized, body coverage is excellent, and wide-beamwidth illumination reduces dark spots in the imagery. Volumetric renderings of this imagery can be obtained using 3-D display software.
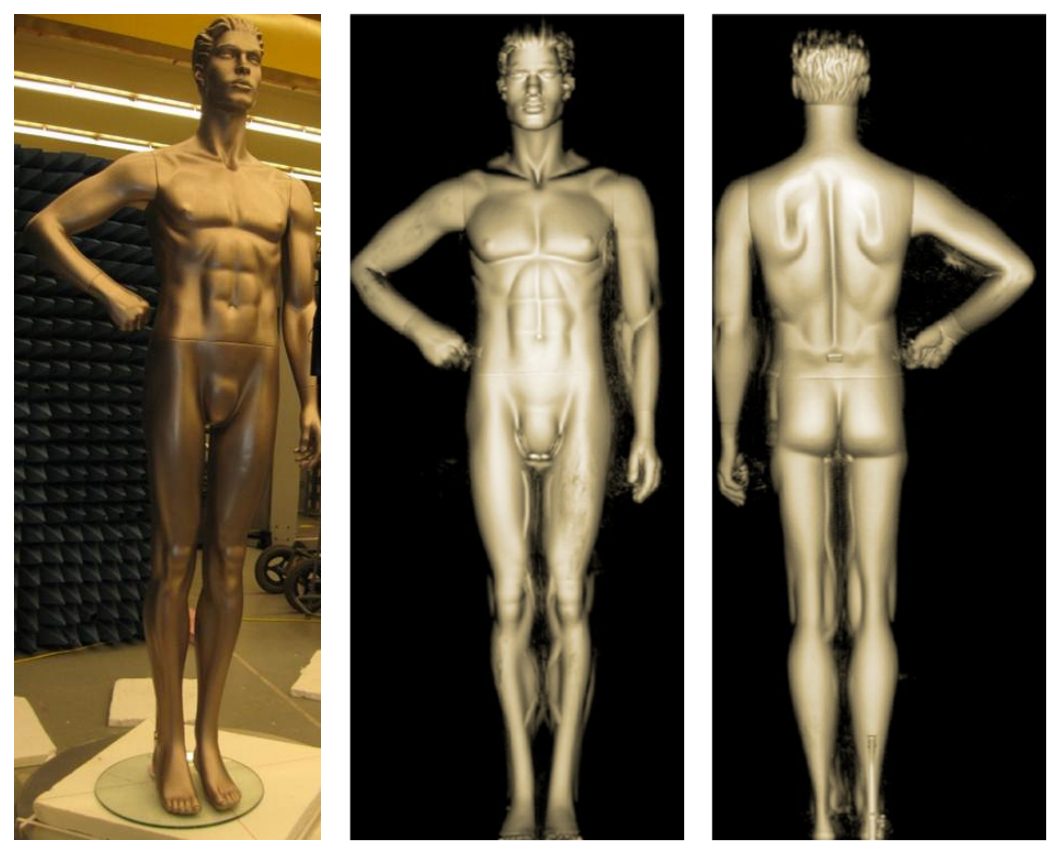

Fig. 8. Experimental $10-40 \mathrm{GHz}$ planar imaging results of a bare mannequin. Photograph of mannequin is shown on the left, with front and side view imaging results shown center and right.

Figure 9 shows a comparison between $10-40 \mathrm{GHz}$ imagery of a full sized mannequin using cylindrical and planer scanning techniques. The cylindrical scan provides 360 degree coverage of the mannequin, but in this figure, only the front section (single frame) of the reconstructed image is shown. Comparing the two images, it is clear that the wide-beamwidth planer scan provides more angular coverage than the cylindrical scanner. However, the cylindrical scan imagery fills in the angular coverage with additional frames from around the body. 

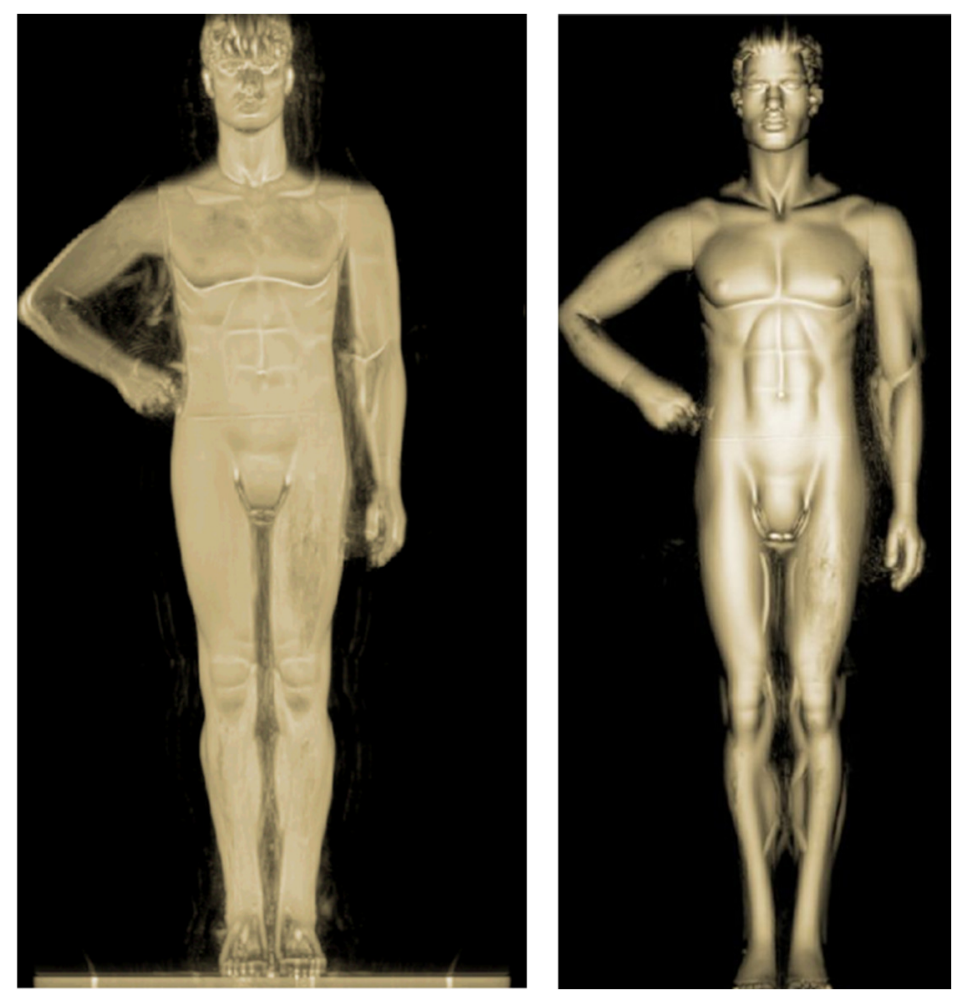

Fig. 9. Experimental $10-40 \mathrm{GHz}$ combined cylindrical imaging results (left) of a bare mannequin compared with the $10-40 \mathrm{GHz}$ wide beamwidth planar imaging results (right).

Depth information from the planer scans can be displayed by using algorithms that define the surface from within the 3-D volume. Figure10 shows an example of a topological rendering of the mannequin that was generated by the VolumeRover software*. This code is freely distributed and use matching cubes algorithm to define the surface from the volumetric imaging data
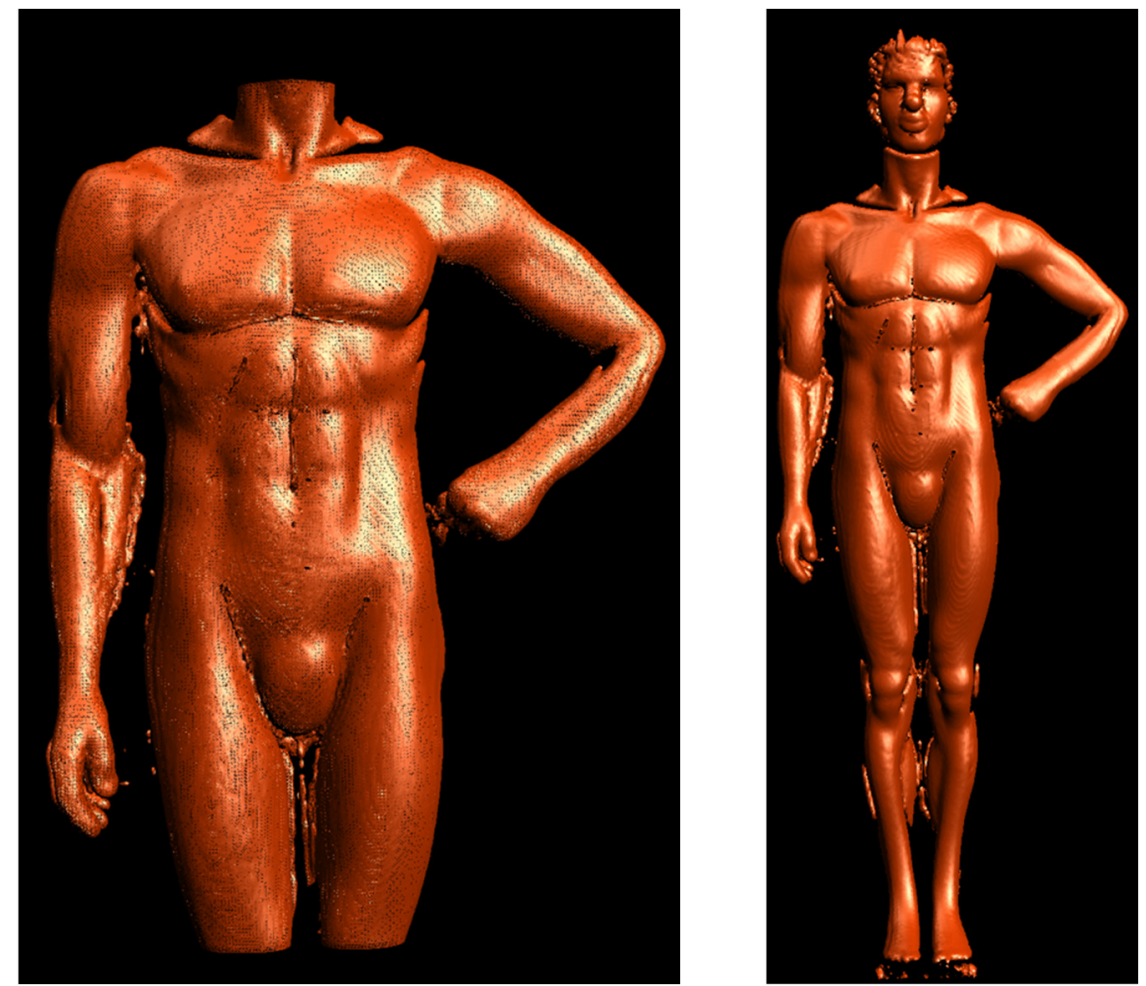

Fig. 10. Rendered surfaces of the $10-40 \mathrm{GHz}$ planar imaging results of the front view of a bare mannequin.

* http://www.cs.utexas.edu/ bajaj/cvcwp/?page id=100 


\section{Conclusions}

First generation millimeter-wave body scanners are commercially available for security and apparel applications. As shown in this paper, PNNL continues to develop and improve this technology. PNNL has developed new technology to improve depth and lateral resolution by using very wide bandwidth transceivers and wide-beamwidth circular polarized antennas. PNNL has developed wide-beamwidth spiral antennas that helps to reduces dark areas in the body scan data and also reduces imaging artifacts from body corner traps and crevices. PNNL has fabricated the next generation millimeter-wave body scanner prototype for the Department of Homeland Security. This system will allow experimental imaging studies to be conducted on people because it will scan fast enough to freeze body motion. This new millimeter-wave body scanner technology can be used for both security and body measurement applications.

\section{Acknowledgements}

Portions of the work described in this paper were conducted with funding from the United States Department of Homeland Security. The Pacific Northwest National Laboratory is operated by Battelle for the U.S. Department of Energy.

\section{References}

[1] http://www.tsa.gov/traveler-information/advanced-imaging-technology-ait

[2] Joanna Gould-Thorpe, VP Technical Operations, Me-Ality, Unique Solutions, Ltd, Question and Answer session at the conclusion of her presentation on "The Power of Aggregate Data;

Gaining Insights and a Competitive Advantage," 3D Body Scanning Technologies Conference, Lugano, Switzerland, 16-17 October 2012.

[3] Elias, B., Airport Body Scanners: The Role of Advanced Imaging Technology in Airline Passenger Screening, Congressional Research Service Report, http://www.fas.org/sgp/crs/homesec/R42750.pdf

[4] McMakin DL, and DM Sheen. 2011. "Millimeter-wave Advanced Imaging Technology" In http://www. aapm.org/meetings/2011AM/. PNWD-SA-9524, Battelle-Pacific Northwest Division, Richland, WA.

[5] Corredoura, P. A., Baharav, Z. A., Taber, B. A. and Lee, G. A., "Millimeter- wave imaging system for personnel screening: Scanning 107 points a second and using no moving parts", Proc. SPIE Passive Millimeter-Wave Imaging Technology IX, vol. 6211, (2006).

[6] Schiessl, A., Genghammer, A., Ahmed, S. S. and Schmidt, L.-P., "Hardware realization of a $2 \mathrm{~m}$ $\mathrm{x} 1 \mathrm{~m}$ fully electronic real-time mm-wave imaging system", Proc. 9th European Conference on Synthetic Aperture Radar (EuSAR), pp. 40-43, (2012).

[7] Sheen DM, DL McMakin, and TE Hall. 2007."Detection of Explosives by Millimeter-wave Imaging." Chapter 9 in Counterterrorist Detection Techniques of Explosives, ed. J Yinon, pp. 237-278. Elsevier B.V., Amsterdam, Netherlands.

[8] Sheen, D.M., et al., Concealed explosive detection on personnel using a wideband holographic millimeter-wave imaging system. Proceedings of SPIE, 1996. 2755: p. 503-13.

[9] Sheen, D.M., D.L. McMakin, and T.E. Hall, Cylindrical millimeter-wave imaging technique for concealed weapon detection. Proceedings of SPIE, 1997. 3240: p. 242-250.

[10] Sheen, D.M., D.L. McMakin, and T.E. Hall, Combined illumination cylindrical millimeter-wave imaging technique for concealed weapon detection. Proceedings of SPIE, 2000. 4032: p. 52-60.

[11] Sheen, D.M., D.L. McMakin, and T.E. Hall, Three-dimensional millimeter-wave imaging for concealed weapon detection. IEEE Transactions on Microwave Theory and Techniques, 2001. 49(9): p. 1581-92.

[12] Sheen, D.M., D.L. McMakin, and T.E. Hall, Cylindrical millimeter-wave imaging technique and applications. Proceedings of SPIE, 2006. 6211.

[13] McMakin DL, DM Sheen, TE Hall, MO Kennedy, and HP Foote. 2007. "Biometric Identification Using Holographic Radar Imaging Techniques." In Sensors, and Command, Control, Communications, and Intelligence (C3I) Technologies for Homeland Security and Homeland Defense VI. Proceedings of SPIE, vol. 6538, ed. Edward M. Carapezza, p. paper no. 65380C .

[14] Sheen, D.M., et al., Circularly polarized millimeter-wave imaging for personnel screening. Proceedings of SPIE, 2005. 5789: p. 117.

[15] Sheen, D.M., J.L. Fernandes, J.R. Tedeschi, D.L. McMakin, A.M . Jones, W.M. Lechelt, and R.H. Severtsen. "Wide-bandwidth, wide-beamwidth, high-resolution, millimeter-wave imaging for concealed weapon detection ", Proc. SPIE 8715, Passive and Active Millimeter-Wave Imaging XVI, 871509 (May 31, 2013); doi:10.1117/12.2016132. 